\title{
Application of scanning focused X- ray imaging positioning technique in preparation of GaAs photocathodes
}

Yijun Zhang, Chengwei Fang, Yunsheng Qian, Junju Zhang, Kaimin Zhang, et al.

Yijun Zhang, Chengwei Fang, Yunsheng Qian, Junju Zhang, Kaimin Zhang, Jingzhi Zhang, Minmin Rong, Feng Shi, Gangcheng Jiao, Xiaofeng Bai, "Application of scanning focused X-ray imaging positioning technique in preparation of GaAs photocathodes," Proc. SPIE 11367, Photosensitive Materials and their Applications, 113671N (1 April 2020); doi: 10.1117/12.2556086

SPIE. Event: SPIE Photonics Europe, 2020, Online Only 


\title{
Application of Scanning focused X-ray imaging positioning technique in preparation of GaAs photocathodes
}

\author{
Yijun Zhang ${ }^{* a}$, Chengwei Fang ${ }^{\mathrm{a}}$, Yunsheng Qian' ${ }^{\mathrm{a}}$, Junju Zhang ${ }^{\mathrm{a}}$, Kaimin Zhang ${ }^{\mathrm{a}}$, Jingzhi Zhang ${ }^{\mathrm{a}}$, \\ Minmin Rong ${ }^{\mathrm{a}}$, Feng Shi ${ }^{\mathrm{b}}$, Gangcheng Jiao ${ }^{\mathrm{b}}$, Xiaofeng Bai ${ }^{\mathrm{b}}$

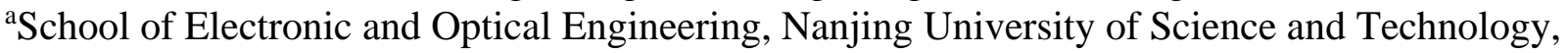 \\ Nanjing 210094, China;

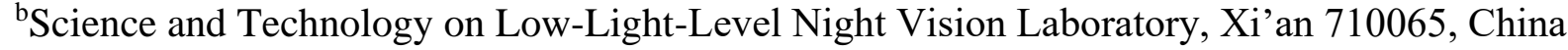

\begin{abstract}
Negative-electron-affinity GaAs-based photocathodes have already found widespread application in modern night vision detectors and vacuum electron sources. Considering the importance of surface micro-area analysis for cathode preparation, a new ultrahigh vacuum interconnection system for photocathode preparation and characterization was developed, wherein the scanning focused X-ray imaging positioning technique combined with the X-ray induced secondary electron image was applied to characterize the surface components in the specified micro region of semiconductor photocathodes. With the aid of the advanced characterization technique, the surface components of micro regions of interest for GaAs cathode samples after cleaning and Cs-O activation were analyzed. The experimental results show that the GaAs cathode samples would be subjected to secondary contamination from the metal sheet of sample holder, accompanied by a small amount of sodium and cesium. The subsequent heat treatment and Cs-O activation can hardly remove the sodium contamination, which can affect the arsenic desorption during heat treatment, hinder the Cs-O adsorption in the activation process, and finally reduce the photoemission performance of the activated cathode. Through the application of the X-ray induced secondary electron image, the optimal cleaning method for GaAs cathode was investigated. This surface characterization technique is of practical value to improving analysis accuracy and optimizing the cathode preparation process.
\end{abstract}

Keywords: GaAs photocathode, scanning focused X-ray imaging positioning, micro region, surface cleanliness, cathode preparation

\section{INTRODUCTION}

Negative electron affinity (NEA) GaAs photocathode has important applications in the optoelectronic fields such as lowlight image intensifiers, transmission electron microscopes, and new solar cells due to its advantages such as high quantum efficiency, small dark current, concentrated energy distribution, and near-infrared response ${ }^{[1-4]}$. Therefore, how to improve the emission performance of GaAs photocathode has been a research issue. It is well known that the cleanliness of the surface has a great influence on the GaAs photocathode emission performance. The surface of GaAs cathode materials is usually contaminated by various impurities, such as carbon pollutions, natural oxides and some inorganic attachments. The presence of impurities will hinder the formation of an effective activation layer on the cathode surface, which will seriously affect the cathode photoemission performance.

The traditional preparation process of GaAs photocathode consists of surface cleaning and cesium oxygen activation ${ }^{[5-7]}$. The commonly used surface cleaning process is divided into two steps: chemical cleaning and high-temperature heating purification. In the preparation of GaAs photocathode, it is usually necessary to use in-situ surface analysis methods in an ultra-high vacuum environment, such as X-ray photoelectron spectroscopy (XPS) to characterize the cleaning and activation effects of the cathode material's surface ${ }^{[6-8]}$. The early XPS belongs to a large-area analysis technology that could not define the area, which results in the inability to understand the pollution distribution on the cathode surface. With the development of the modern XPS technology, the scanning focused X-ray light source can be used to reduce the analysis area to the micron level. The combination of the scanning focused X-ray imaging positioning technique and the

*zhangyijun423@126.com; phone 86 02584303075; fax 8602584303075

Photosensitive Materials and their Applications, edited by Robert R. McLeod, Inmaculada Pascual Villalobos,

Yasuo Tomita, John T. Sheridan, Proc. of SPIE Vol. 11367, 113671N · C 2020 SPIE

CCC code: $0277-786 \mathrm{X} / 20 / \$ 21 \cdot$ doi: $10.1117 / 12.2556086$ 
X-ray Induced Secondary Electron Image (SXI) has greatly improved the accuracy of surface detection ${ }^{[9,10]}$. Therefore, how to apply advanced XPS technology to photocathode preparation requires further investigation.

In this paper, the function of quasi-in-situ surface analysis of reflective photocathodes was realized by using the ultrahigh vacuum interconnection system for photocathode preparation and characterization (UHVIS-PPC). The SXI images were generated by scanning focused X-rays to excite the surface of the reflective GaAs photocathode samples, and then the surface micro-area characteristics were analyzed based on the SXI image to accurately detect the chemical elements on the surface. Based on the scanning focused X-ray imaging technology, the elements in the micro region of GaAs cathode surface after chemical cleaning and cesium/oxygen activation were analyzed respectively, and the possible contamination of sample metal sheet to the cathode sample surface after chemical cleaning was explored, and the influence of different surface treatment methods on the surface cleanliness and activation properties were further studied. Through the combination of ultra-high vacuum interconnecting technology and surface analysis technology to study the effect of surface contaminants on the cathode activation process and quantum efficiency.

\section{EXPERIMENTAL}

In the experiment, p-type reflective GaAs photocathode with (100)-oriented surface was used, the doping concentration is $1 \times 10^{19} \mathrm{~cm}^{-3}$, the thickness is $300 \mu \mathrm{m}$. All the samples with the area of $11 \mathrm{~mm} \times 11 \mathrm{~mm}$ used were cleaved from the same wafer with a diameter of $50.8 \mathrm{~mm}$. Three samples were selected and named as sample 1, sample 2 and sample 3 , respectively. In the process of chemical cleaning, the samples were first subjected to surface degreasing treatment, the samples were accordingly immersed in carbon tetrachloride, acetone, ethanol, and deionized water, and ultrasonically cleaned for $5 \mathrm{~min}$ respectively. Then the sample 1 and 2 were chemically etched using an wet cleaning method that HF acid solution $(40 \%)$ for 5 minutes + HCl: IPA mixed solution (1:10) cleaning for 5 minutes, whereas, the sample 3 underwent the $\mathrm{UV}-\mathrm{O}_{3}$ cleaning before the chemical cleaning. After the above cleaning steps were completed, the samples were repeatedly washed with deionized water and dried with nitrogen. After the chemical cleaning treatment, the samples needed to be fixed on the sample holder as shown in Fig. 1(a). Sample 1 was fixed with the metal sheet that had not been cleaned after previous activation experiments. However, sample 2 and 3 were fixed with the metal sheet had been cleaned by ultrasonic cleaning with acetone, ethanol, and deionized water. After introduced into the loading chamber, the samples were transmitted to the ultra-high vacuum system using a magnetic transfer arm. The schematic diagram of the experimental system is shown in Fig. 1(b), which is the ultra-high vacuum connection of the sample loading chamber, pretreatment chamber, activation chamber and surface analysis chamber, and the multi-information online measurement and control system is used to complete the cathode activation experiment. The XPS analysis system in the interconnection setup is PHI5000 VersaProbe II produced by ULVAC-PHI, which uses a monochromatic aluminum target $(\mathrm{Al}-\mathrm{K} \alpha)$ scanning focused $\mathrm{X}$-ray source $(h v=1486.6 \mathrm{eV})$. The excited photoelectron process is as follows: the focused electron gun is used to scan the $\mathrm{Al}$ anode target, and the $\mathrm{X}$-ray generated is reflected by the monochromatic focused quartz crystal, and then irradiated onto the sample surface for scanning with the electron beam as a mirror image, so as to realize the X-ray photoelectron spectroscopy analysis of the micro-area on the sample surface. The X-ray beam spot diameter is continuously adjustable from 10 to $400 \mu \mathrm{m}$, a $50 \mu \mathrm{m}$ X-ray beam spot diameter was used for micro-area analysis in this paper. The pass energy is $58.7 \mathrm{eV}$, the energy step is $0.125 \mathrm{eV}$, and the background vacuum is better than $6 \times 10^{-8} \mathrm{~Pa}$.

SXI image was generated by X-rays excitation on the surface of the sample after chemical cleaning by using scanning focused X-ray imaging positioning technology. The chemical compositions in micro-area of the cathode surface were analyzed and detected, and then the sample was transmitted to the ultra-high vacuum activation chamber for heat treatment of $600^{\circ} \mathrm{C}$ to remove the carbon and oxygen pollutants on the surface. After thermal purification, the sample was activated by cesium (Cs) and oxygen $(\mathrm{O})$ to lower the surface work function and form NEA surface, thereby improving the escape probability of photoelectrons. The $\mathrm{Cs}$ and $\mathrm{O}$ activation technology adopted the Cs source continuous and $\mathrm{O}$ source intermittent activation method ${ }^{[11]}$. The background vacuum degree of the activation chamber was better than $3 \times 10^{-8} \mathrm{~Pa}$. During activation, the surface of the cathode sample was illuminated with white light from a halogen lamp, the photocurrent and the quantum efficiency curve after the activation were tested in-situ by the multiinformation on-line measurement and control system. After activation, the sample was transmitted to the XPS analysis system again for surface chemical composition detection. In the process of sample transfer between the activation chamber and the surface analysis chamber, the vacuum degree was better than $5 \times 10^{-8} \mathrm{~Pa}$, and each sample transport process consumed less than $5 \mathrm{~min}$, which avoided the possibility of sample surface recontamination. 


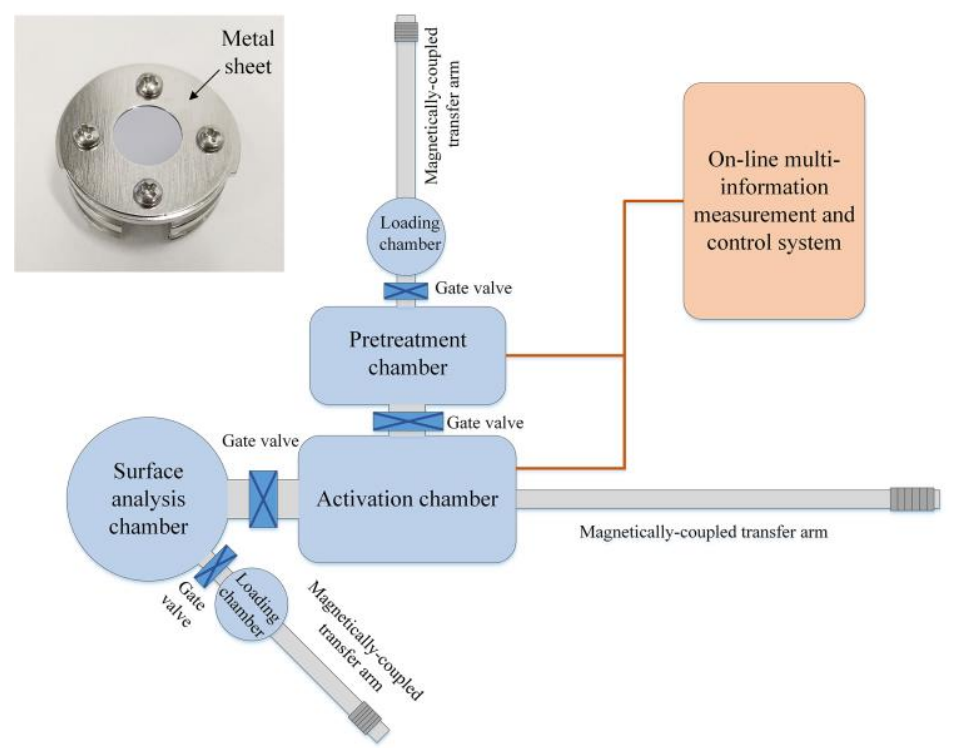

Figure 1. Experimental sample stage and diagram of UHV interconnection setup.
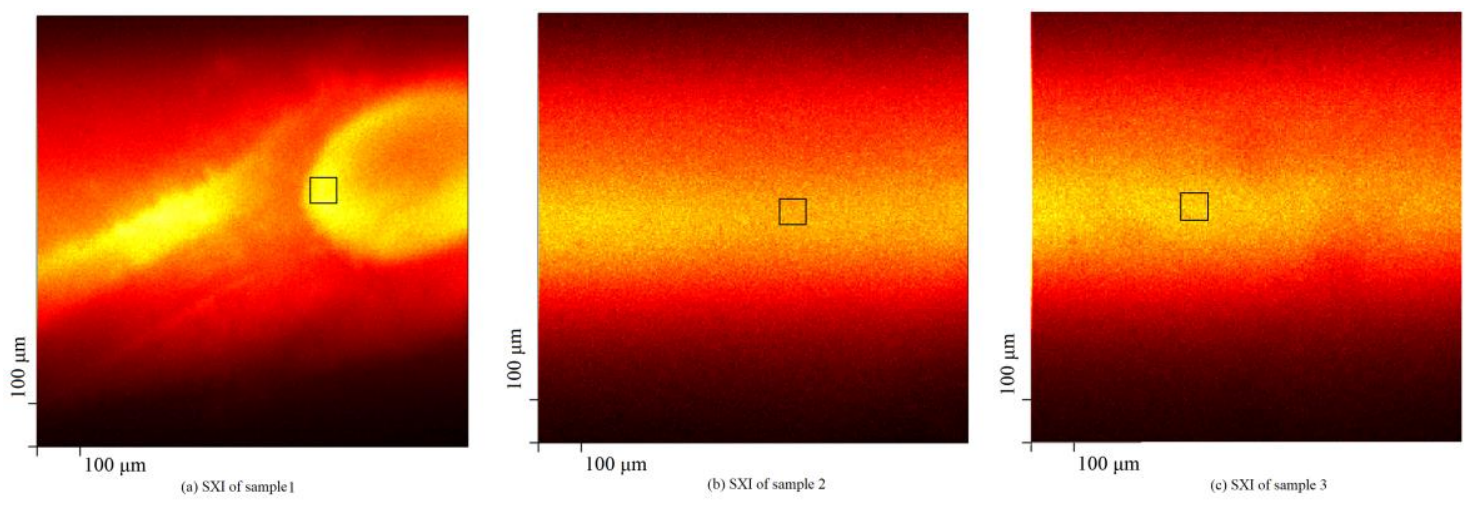

Figure 2. SXI photos for the three samples after the chemical cleaning process.

\section{RESULTS AND ANALYSIS}

\subsection{XPS analysis}

The GaAs cathode sample after chemical cleaning was sent into the vacuum system, the scanning focused monochromatic X-ray was used to perform micro-area selection analysis. First, the sample was excited by X-ray to generate an SXI image, as shown in Fig. 2. It can be clearly seen that the surface of sample 1 showed an irregularly shaped impurity plaque distribution, while the surface of sample 2 and 3 were distributed in a center-symmetric band, without impurity plaque distribution. With the aid of the scanning focused X-ray imaging positioning technique combined with the SXI image function, the plaque area of interest in the SXI image was selected labeled as the black box as shown in Fig. 2, and the $50 \mu \mathrm{m} \mathrm{X}$-ray beam spot was used to accurately analyze photoelectron spectroscopy in the box area.

After the sample was activated, XPS analysis was performed again in the same area of the activated sample surface. The detected surface chemical composition of the three samples after chemical cleaning and activation are shown in Table 1. It can be seen from Table 1 that compared with the two other samples, the impurity plaques in sample 1 not only contain more $\mathrm{C}$ and $\mathrm{O}$ impurity pollution, but also $\mathrm{Na}$ and $\mathrm{Cs}$ pollution, which were not found in the previous XPS test, indicating that if the metal sheet used to fix the cathode sample is not thoroughly cleaned after multiple activations, it 
will easily contaminate the cleaned cathode sample when used repeatedly. It can be seen that $\mathrm{Na}$ contamination existed on the surface of sample 1 after chemical cleaning and activation, which shows that the Na pollution on the cathode surface could not be removed by heating purification at $600^{\circ} \mathrm{C}$ or Cs/O activation. In Table 1, there is a small amount of Cs $3 \mathrm{~d}$ signal after sample 1 was chemically cleaned, and no Cs $3 \mathrm{~d}$ peak signal was detected before samples 2 and 3 was activated. Due to the adsorption of Cs in the process of activation, Cs 3d appeared on the surfaces of samples 1 and 2. The XPS signal of $\mathrm{C} 1 \mathrm{~s}$ and $\mathrm{O} 1 \mathrm{~s}$ of sample 1 are higher than those of samples 2 and 3 . In addition, the elemental contents of $\mathrm{Ga} 3 \mathrm{~d}$ and $\mathrm{As} 3 \mathrm{~d}$ on the surface of sample 1 is much lower than those of samples 2 and 3, which indicates that the surface Na pollution and other impurities such as $\mathrm{C}$ and $\mathrm{O}$ will affect the heat purification effect. By comparison of XPS spectra between sample 2 and sample 3, it can be seen that after the chemical cleaning, the $\mathrm{C} 1 \mathrm{~s}$ content of sample 3 is less than that of sample 2, but the $\mathrm{O} 1$ s content is greater than that of sample 2 due to the $\mathrm{UV}-\mathrm{O}_{3}$ treatment after chemical cleaning. After activation, there is still a little $\mathrm{C}$ component on the surface of sample 2, and the $\mathrm{O} 1 \mathrm{~s}$ content of sample 2 exceeds that of sample 3. However, the content ratio of $\mathrm{Cs}$ and $\mathrm{O}$ is close to 2:1 for sample 3 .

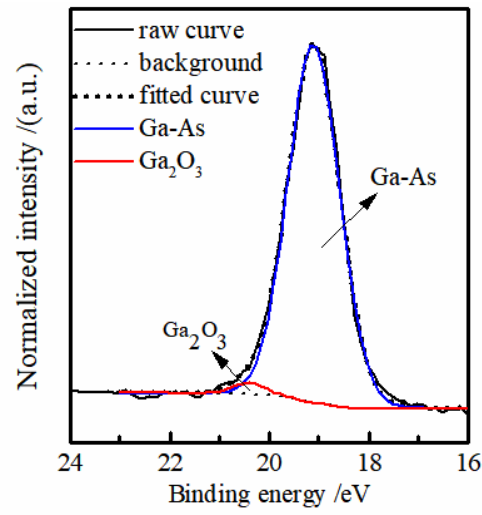

(a) Sample 1 after chemical cleaning

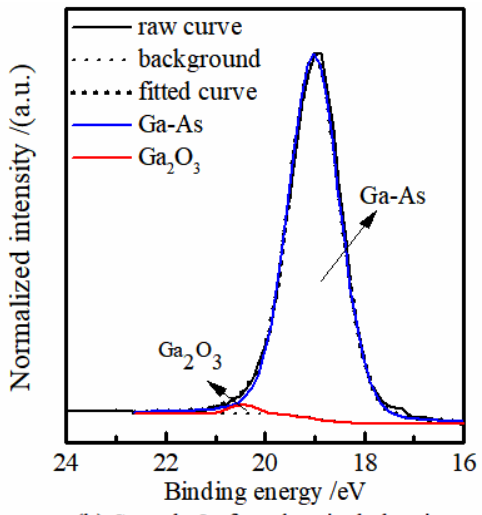

(b) Sample 2 after chemical cleaning

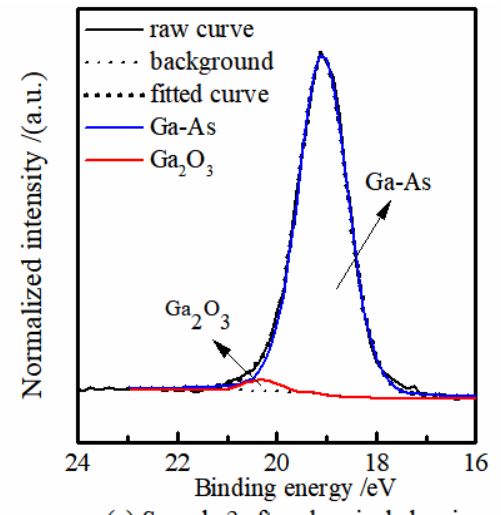

(c) Sample 3 after chemical cleaning

Figure 3. XPS spectra of Ga 3d after chemical cleaning for the three samples.

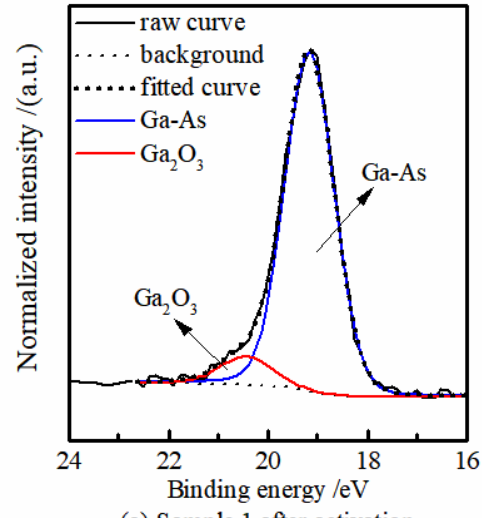

(a) Sample 1 after activation

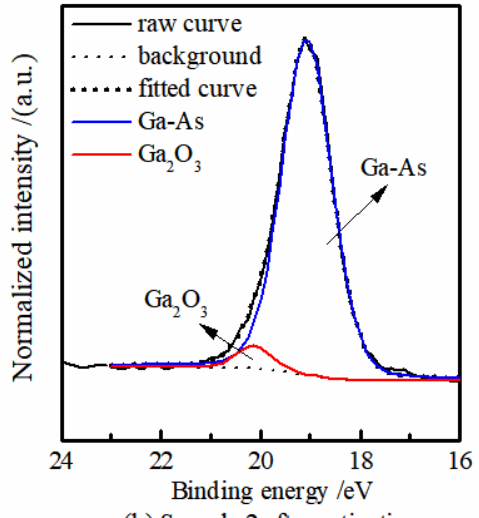

(b) Sample 2 after activation

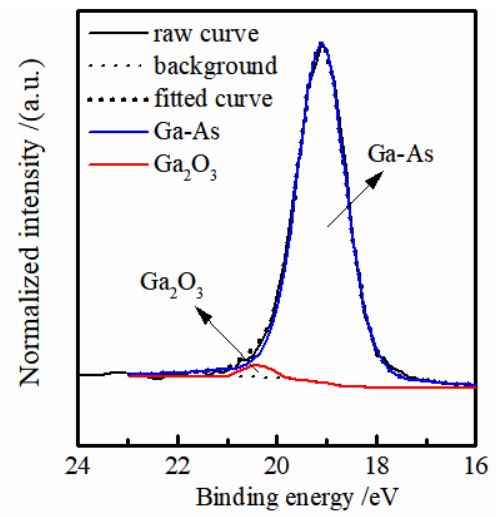

(c) Sample 3 after activation

Figure 4. XPS spectra of Ga $3 d$ after activation for the three samples.

Figure 3 shows the XPS spectra and peak fitting spectra of Ga $3 \mathrm{~d}$ on the surface of samples after chemical cleaning. The normal Shirley-style background line and Gauss-Lorentz function are used in the peak fitting. It can be seen from Fig. 3 that the peaks of $\mathrm{Ga} 3 \mathrm{~d}$ on the sample has two states, namely $\mathrm{Ga}_{2} \mathrm{O}_{3}$ with a high binding energy peak $(20.4 \mathrm{eV})$ and $\mathrm{GaAs}$ bulk with a low binding energy peak $(19.1 \mathrm{eV})$. The calculated oxide percentages are shown in Table 2 . After chemical cleaning, $\mathrm{Ga}_{2} \mathrm{O}_{3}$ in samples 1,2 and 3 accounted for $2.08 \%, 1.21 \%$ and $2.36 \%$ of the total amount of Ga $3 \mathrm{~d}$, respectively, indicating that the chemical cleaning had a significant removal effect on the surface natural oxide layer. Because of the additional UV-ozone treatment, the oxide content of Ga $3 \mathrm{~d}$ on sample 3 is the highest. Figure 4 shows the XPS spectra and split peak fitting spectra of $\mathrm{Ga} 3 \mathrm{~d}$ after activation. The proportion of $\mathrm{Ga}_{2} \mathrm{O}_{3}$ in the whole $\mathrm{Ga} 3 \mathrm{~d}$ spectrum increased due to the adsorption of $\mathrm{O}$ during the activation. From Table 2, it can be seen that $\mathrm{Ga}_{2} \mathrm{O}_{3}$ in sample 1 of Fig. 4(a) accounts for $8.11 \%$ of the total $\mathrm{Ga} 3 \mathrm{~d}, \mathrm{Ga}_{2} \mathrm{O}_{3}$ in sample 2 of Fig. 4(b) accounts for $4.62 \%$ of the total $\mathrm{Ga} 3 \mathrm{~d}$, and that 
ratio of sample 3 is $1.92 \%$. The change in $\mathrm{Ga}_{2} \mathrm{O}_{3}$ content indicates that the difference in the proportion of $\mathrm{Ga}_{2} \mathrm{O}_{3}$ to $\mathrm{Ga}_{3} \mathrm{~d}$ among the three samples was obvious. It seems that the presence of $\mathrm{Na}$ and $\mathrm{Cs}$ pollution and residual $\mathrm{C}$ impurities on the surface can promote the adsorption of $\mathrm{O}$ on the activated $\mathrm{Ga}$ atom. This may be due to the small electronegativity of $\mathrm{Ga}$, which is more likely to react with the more electronegative adsorbed $\mathrm{O}$ to generate oxides ${ }^{[12]}$.

Table 1. Surface chemical compositions and contents in the selected regions of SXI photos for the three samples.

\begin{tabular}{|c|c|c|c|c|c|c|}
\hline Samples & C 1s (\%) & O 1s (\%) & Ga 3d (\%) & As 3d (\%) & Na 1s (\%) & Cs 3d (\%) \\
\hline $\begin{array}{c}\text { Sample 1 after } \\
\text { chemical cleaning }\end{array}$ & 35.11 & 24.53 & 11.12 & 14.08 & 12.51 & 2.65 \\
\hline $\begin{array}{c}\text { Sample 2 after } \\
\text { chemical cleaning }\end{array}$ & 8.81 & 7.30 & 38.56 & 45.33 & 0.00 & 0.00 \\
\hline $\begin{array}{c}\text { Sample 3 after } \\
\text { chemical cleaning }\end{array}$ & 5.06 & 9.36 & 39.94 & 45.64 & 0.00 & 0.00 \\
\hline $\begin{array}{c}\text { Sample 1 after } \\
\text { activation }\end{array}$ & 10.78 & 33.86 & 9.74 & 9.91 & 14.91 & 20.80 \\
\hline $\begin{array}{c}\text { Sample 2 after } \\
\text { activation }\end{array}$ & 2.85 & 15.59 & 35.34 & 24.50 & 0.00 & 21.72 \\
\hline $\begin{array}{c}\text { Sample 3 after } \\
\text { activation }\end{array}$ & 0.00 & 9.16 & 40.60 & 31.38 & 0.00 & 18.86 \\
\hline
\end{tabular}

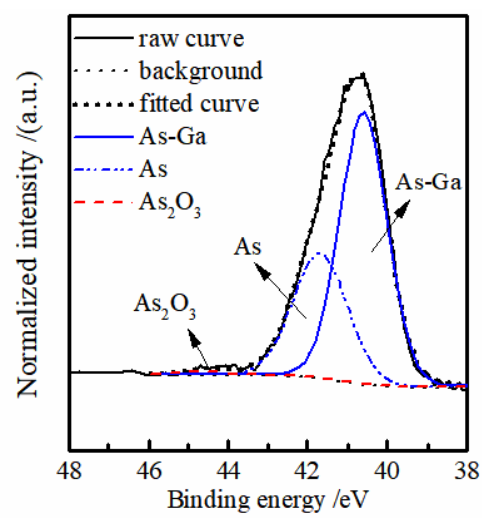

(a) Sample 1 after chemical cleaning

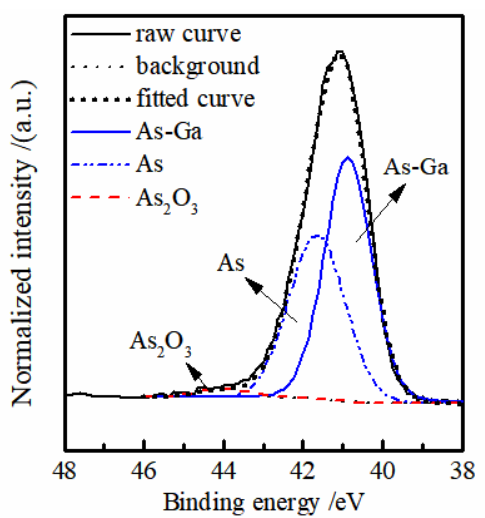

(b) Sample 2 after chemical cleaning

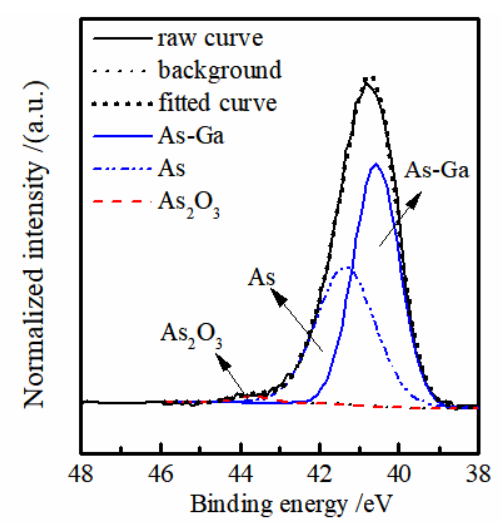

(c) Sample 3 after chemical cleaning

Figure 5. XPS spectra of As 3d after chemical cleaning for the three samples.

Figure 5 and Figure 6 shows the XPS spectra and split peak fitting spectra of As $3 \mathrm{~d}$. The As $3 \mathrm{~d}$ peaks in the sample can be fitted to the spectrum peaks in three states, from the high binding energy end to the low binding energy end $\operatorname{are} \mathrm{As}_{2} \mathrm{O}_{3}$ (44.4ev), As (41.6ev) and GaAs bulk (40.8ev) respectively. It can be seen from Table 2 that the ratio of the peaks of sample 1 in the three states of $\mathrm{As}_{2} \mathrm{O}_{3}$, As and $\mathrm{GaAs}$ bulk after chemical cleaning is $0.93 \%, 34.30 \%$ and $64.77 \%$, respectively, while the ratio of the peaks after activation is $1.75 \%, 31.62 \%$ and $66.63 \%$, which shows that the three peaks of sample 1 in As 3d spectrum were not changed very much. For sample 2, the ratio of $\mathrm{As}_{2} \mathrm{O}_{3}$ 、 As and GaAs after chemical cleaning are $0.97 \%, 43.21 \%$, and $55.82 \%$, and the peaks after activation are $0 \%$ and $33.27 \%$ and $66.73 \%$. While for sample 3, the ratio of $\mathrm{As}_{2} \mathrm{O}_{3}$, As and GaAs after chemical cleaning are $0.99 \%, 43.01 \%$, and $56 \%$, and the peaks after activation are $0 \%$ and $29.93 \%$ and $70.07 \%$. It can be clearly seen that the bulk GaAs peak proportion in samples 2 and 3 increased, while the As spectral peak proportion decreased significantly. Besides, the $\mathrm{As}_{2} \mathrm{O}_{3}$ component disappeared after activation for samples 2 and 3, which is because that during heating purification, $\mathrm{As}_{2} \mathrm{O}_{3}$ can react with $\mathrm{GaAs}$ to generate volatile $\mathrm{As}^{[13,14]}$. Furthermore, it is found that the proportion of As peak and $\mathrm{As}_{2} \mathrm{O}_{3}$ peak in sample 1 has little change before and after activation, which is mainly because $\mathrm{Na}$ and other impurities hindered the thermal volatilization of As and conversely enhanced the reaction between As and adsorbed O. From the results in Table 2, it can be inferred that for the original $\mathrm{GaAs}$ photocathode without $\mathrm{Na}$ and $\mathrm{Cs}$ pollutants, in addition to the $\mathrm{Cs}-\mathrm{O}$ dipoles formed 
on the surface of the active cathode, there exist extra Ga oxides. Therefore, the surface of the GaAs photocathode

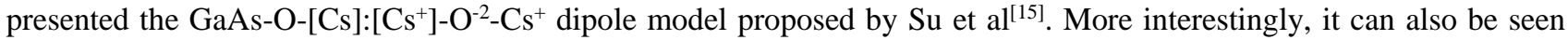
from Table 1 that the proportion of $\mathrm{O}$ content in the activated surface of sample 3 is minimum. Whether the impurity pollutions such as $\mathrm{Na}$, $\mathrm{Cs}$ and $\mathrm{C}$ affect the removal of original oxides during heat treatment and the following $\mathrm{O}$ adsorption during activation needs to be further studied.

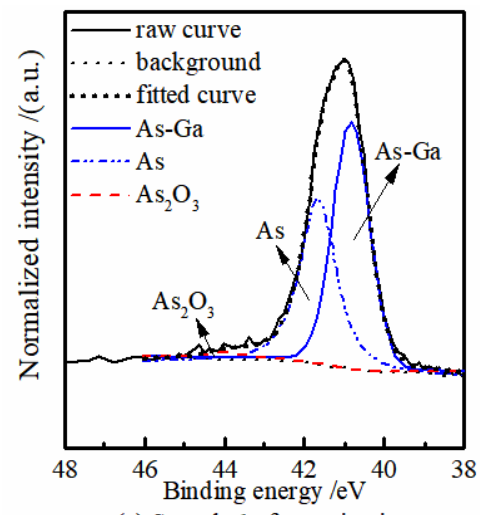

(a) Sample 1 after activation

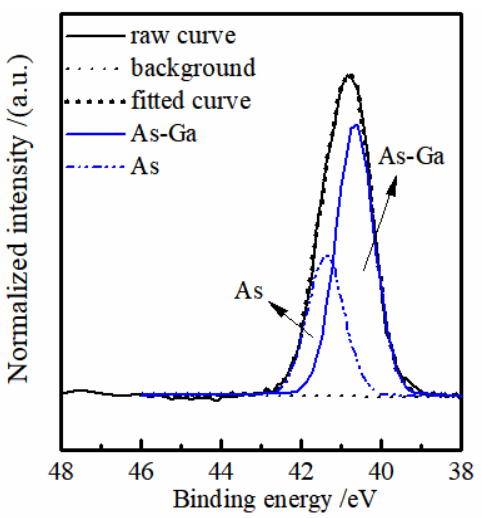

(b) Sample 2 after activation

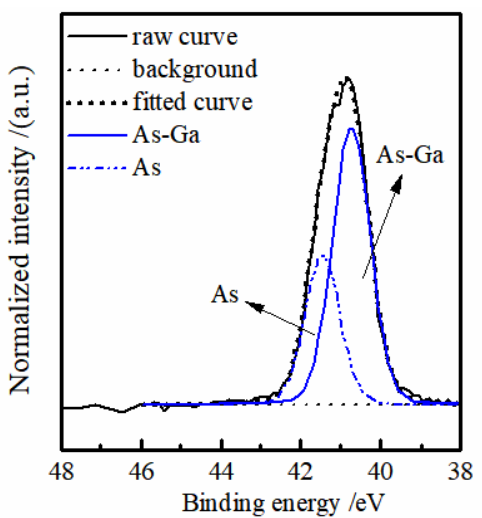

(c) Sample 3 after activation

Figure 6. XPS spectra of As 3d after activation for the three samples.

Table 2. XPS fitted peak ratios of Ga $3 \mathrm{~d}$ and As $3 \mathrm{~d}$ after chemical cleaning and activation for the three samples.

\begin{tabular}{|c|c|c|c|c|c|}
\hline Samples & \multicolumn{2}{|c|}{ Ga 3d (\%) } & \multicolumn{3}{c|}{ As 3d (\%) } \\
As & As-Ga & \multicolumn{2}{c|}{$\mathbf{A s}_{2} \mathbf{O}_{3}$} \\
\hline $\begin{array}{c}\text { Sample 1 after chemical } \\
\text { cleaning }\end{array}$ & 97.92 & 2.08 & 64.77 & 34.30 & 0.93 \\
\hline $\begin{array}{c}\text { Sample 2 after chemical } \\
\text { cleaning }\end{array}$ & 98.79 & 1.21 & 55.82 & 43.21 & 0.97 \\
\hline $\begin{array}{c}\text { Sample 3 after chemical } \\
\text { cleaning }\end{array}$ & 97.64 & 2.36 & 56.00 & 43.01 & 0.99 \\
\hline $\begin{array}{c}\text { Sample 1 after activation } \\
\text { Sample 2 after activation }\end{array}$ & 91.89 & 8.11 & 66.63 & 31.62 & 1.75 \\
\hline Sample 3 after activation & 98.08 & 4.62 & 66.73 & 33.27 & 0.00 \\
\hline
\end{tabular}

\subsection{Activation analysis}

In addition to the XPS analysis of the surface chemical composition of the cleaned and activated GaAs photocathode samples, it is also necessary to directly determine the effect of metal contamination and different cleaning methods on cathode activation performance through activation experiments. Using the multi-information on-line measurement and control system, the photocurrent curves of the GaAs cathode samples activated by $\mathrm{Cs}$ and $\mathrm{O}$ co-deposition recorded in real time are shown in Fig. 7. It can be seen from the comparison that the first Cs peak of sample 3 is the earliest and the final photocurrent is the largest. Although the rise time of photocurrent curve of sample 2 is slightly slower than that of sample 3, the increase extent of photocurrent is not so large, and the final photocurrent peak is smaller than that of sample 3. The photocurrent appears in sample 1 after activation for about 15 minutes, and the first Cs peak rise slowly, and the final photocurrent peak is the smallest. Therefore, the atomically clean surface of the GaAs photocathode is very important for the $\mathrm{Cs}$ and $\mathrm{O}$ adsorption, which is conducive to generating a larger photocurrent, which is well proved by the activation phenomena. 


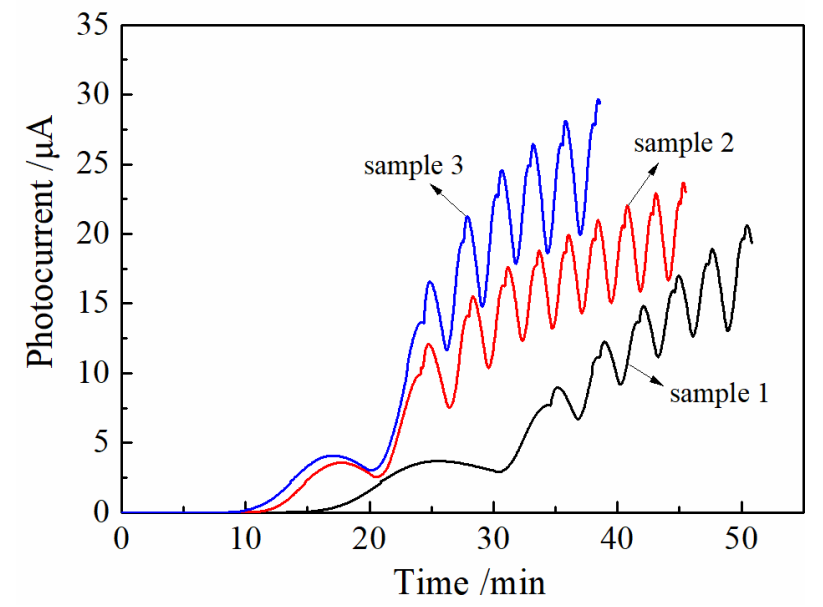

Figure 7. Photocurrent curves of the three samples.

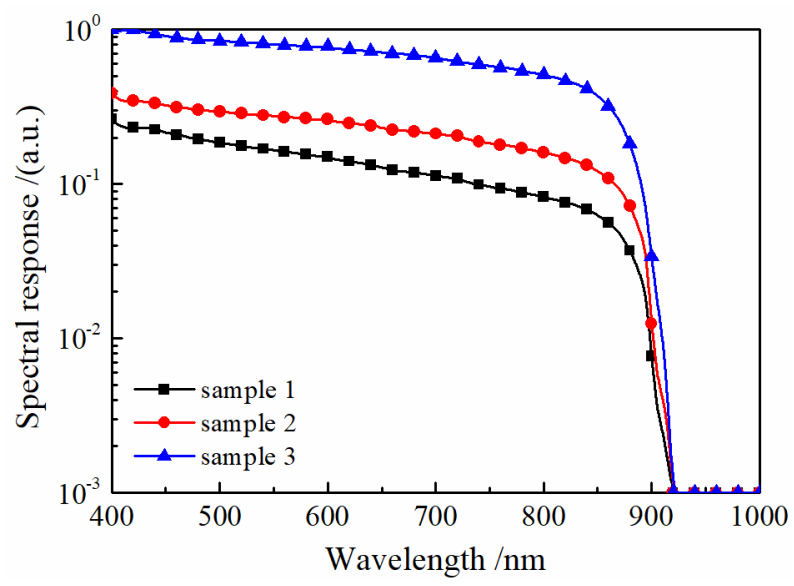

Figure 8. Spectral response curves of the three samples.

Through the on-line spectral response test system, the quantum efficiency curves of the activated samples were also measured, as shown in Fig. 8. It can be seen from the figure that the spectral response of sample 3 is significantly greater than that of sample 1 and sample 2 at the same wavelength, and sample 3 has higher quantum efficiency in the whole band range. Since the three samples were all from the same GaAs photocathode single crystal wafer, they had the same structure and photoelectric physical properties. Therefore, one of the important factors that cause the difference of the emission performance between the three is the difference of the surface electron affinity, which is determined by the surface potential barrier. The photoelectrons of the GaAs cathode are excited by the incident photons, and the photoelectrons will be scattered by the surface barrier during the escape to the surface. The impurity contamination on the surface is obviously not conducive to the well-ordered adsorption of $\mathrm{Cs}$ and $\mathrm{O}$ and the formation of double dipole layers, which will cause a larger surface barrier and result in the overall low quantum efficiency of the whole band.

\section{CONCLUSION}

In this paper, the quasi-in-situ surface analysis function of reflective GaAs cathode samples before and after activation were realized by using the ultra-high vacuum interconnection setup for photocathode preparation and surface analysis. With the aid of scanning focused XPS secondary electronic imaging and micro-area analysis, it is found that the GaAs cathode after chemical cleaning would be subject to secondary contamination from metal sheet of fixed samples, resulting in surface contaminations such as $\mathrm{Na}, \mathrm{Cs}, \mathrm{C}$ and other impurities, and high-temperature purification and $\mathrm{Cs} / \mathrm{O}$ activation did not remove $\mathrm{Na}$ contamination present on the GaAs surface, and surface contaminations would hinder the thermal volatilization of As and enhance the formation of $\mathrm{As}_{2} \mathrm{O}_{3}$ during activation. Meanwhile, the surface of GaAs 
treated by different cleaning methods had also been characterized. The activation results showed that the presence of surface contaminations would affect the adsorption of $\mathrm{Cs}$ and $\mathrm{O}$ during the activation process, which was not conducive to the formation of an effective dipole layer and reduced the photoelectric emission performance of the GaAs cathode. This contamination can be completely avoided by effectively cleaning the metal sheet and adding $\mathrm{UV}-\mathrm{O}_{3}$ treatment in wet cleaning. The advanced scanning focused X-ray imaging positioning technique can more accurately detect the chemical element composition on the micro-area surface of interest, which has important application value for optimizing the preparation process and improving the cathode performance.

\section{ACKNOWLEDGMENTS}

This work was supported by the National Natural Science Foundation of China (Grant Nos. 61771245 and 61301023), and Science and Technology on Low-Light-Level Night Vision Laboratory Foundation of China (Grant No. J20150702).

\section{REFERENCES}

[1] Chrzanowski, K., "Review of night vision technology," Opto-electronics Review. 21(2), 153-181 (2013).

[2] Zhang, Y. J., Niu, J., Zhao, J., Zou, J. J., and Chang, B. K., "Effect of exponenial-doping structure on quantum yield of transmission-mode GaAs photocathodes," Acta Physica Sinica. 60(6), 067301 (2011).

[3] Kuwahara, M., Kusunoki, S., Nambo, Y., Saitoh, K., Jin, X., Ujihara, T., Asano, H., Takeda, Y., and Yanaka, N., "Coherence of a spin-polarized electron beam emitted from a semiconductor photocathode in a transmission electron microscope," Applied Physics Letters, 105(19), 193101 (2014).

[4] Yang, Y., Yang, W., and Sun, C., "Heterostructured cathode with graded bandgap window-layer for photonenhanced thermionic emission solar energy converters," Solar Energy Materials \& Solar Cells, 132, 410-417 (2015).

[5] Feng, C., Zhang, Y. J., Liu, J., Qian, Y. S., Zhang, J. Z., Shi, F., and Bai, X. F., "Optimized chemical cleaning procedure for enhancing photoemission from GaAs photocathode," Materials Science in Semiconductor Processing, 91, 41-46 (2019).

[6] Liu, Z., Sun, Y., Machuca, F., Pianetta, P., and Pease, F., "Preparation of clean GaAs (100) studied by synchrotron radiation photoemission," Journal of Vacuum Science \& Technology A, 21(1), 212-218 (2003).

[7] Tereshenko, O. E., Chikichev, S. I., and Terekhov, A. S., "Composition and structure of HCl-isopropanol treated and vacuum annealed GaAs(100) surfaces," Journal of Vacuum Science \& Technology A, 17(5), 26552662 (1999).

[8] Zou, J. J., Chang, B. K., Yang, Z., Zhang, Y. J., and Qiao, J. L., "Evolution of surface potential barrier for negative-electron-affinity GaAs photocathodes," Journal of Applied Physics, 105, 013714 (2009).

[9] Friedman, A. K., Wenqing, S., Yaroslav, L., Siedle, A. R., and Baker, L. A., "Mapping microscale chemical heterogeneity in Nafion membranes with X-ray photoelectron spectroscopy," Journal of The Electrochemical Society, 165(11), H733-H741 (2018).

[10] Moulser, J. F., "The impact of the scanning XPS microprobe on industrial applications of X-ray photoelectron spectroscopy," Journal of Electron Spectroscopy \& Related Phenomena, 231, 43-49 (2019).

[11]Zhang, Y. J., Qian, Y. S., Feng, C., Shi, F., Cheng, H. C., and Zou, J. J., "Improved activation technique for preparing high-efficiency GaAs photocathodes," Optical Materials Express, 7(9), 3456-3465 (2017).

[12] Li, X. F., Zhang, J. W., Gao, H. K., and Hou, X., "Surface XPS analysis of transparent GaAs photocathode oxidized in vacuum baking," Acta Photonica Sinica, 31(6), 778-780 (2002).

[13] Pun, A. F., Wang, X., Durbin, S. M., and Zheng, J. P., "Reduction of thermal oxide desorption etching on gallium arsenide," Thin Solid Films, 515(10), 4419-4422 (2007).

[14] Yamada, M., and Ide, Y., "Anomalous behaviors observed in the isothermal desorption of GaAs surface oxides," Surface Science, 339(339), L914-L918 (1995).

[15]Su, C. Y., Spicer, W. E., and Lindau, I., "Photoelectron spectroscopic determination of the structure of (Cs, O) activated GaAs (110) surfaces," Journal of Applied Physics, 54(3), 1413-1422 (1983). 